\title{
Pairing in two-dimensional boson-fermion mixtures
}

\author{
J. Mur-Petit and A. Polls \\ Departament d'Estructura i Constituents de la Matèria, Universitat de Barcelona, Avenida Diagonal 647, E-08028 Barcelona, Spain \\ M. Baldo and H.-J. Schulze \\ Sezione INFN, Dipartimento di Fisica, Università di Catania, Via Santa Sofia 64, I-95123 Catania, Italy \\ (Received 11 August 2003; published 9 February 2004)
}

\begin{abstract}
The possibilities of pairing in two-dimensional boson-fermion mixtures are carefully analyzed. It is shown that the boson-induced attraction between two identical fermions dominates the $p$ wave pairing at low density. For a given fermion density, the pairing gap becomes maximal at a certain optimal boson concentration. The conditions for observing pairing in current experiments are discussed.
\end{abstract}

DOI: 10.1103/PhysRevA.69.023606

PACS number(s): $03.75 . \mathrm{Ss}, 03.75 . \mathrm{Nt}, 74.20 . \mathrm{Fg}$

Since the first realization of a degenerate Fermi gas [1], the search for a BCS-like transition signature in ultracold trapped gases of fermionic atoms has received a lot of attention both from theoretical and experimental points of view. Already before the experimental achievement of Ref. [1], there had been suggestions on the possibility to observe this transition in a gas with two hyperfine components of ${ }^{6} \mathrm{Li}$ [2]. The importance of the asymmetry in the populations of the two components was studied in Refs. [3,4]. Later on, the influence of adding bosons and the presence of a BoseEinstein condensate (BEC) on the transition temperature of the Fermi gas was studied for a three-dimensional trap in Refs. [5-7].

At the same time, the possibility to design the trap so as to produce effectively one- and two-dimensional systems has attracted much interest in theoretically describing [8-10] and experimentally obtaining [11] such low-dimensional quantum systems, where correlations play generally a more important role than in their three-dimensional (3D) counterparts.

In this paper we discuss the principal features of pairing in a very dilute two-dimensional mixture of fermions and bosons, characterized by their masses $m_{F}$ and $m_{B}$; densities $\rho_{F}=k_{F}^{2} / 4 \pi$ and $\rho_{B}$; and chemical potentials $\mu_{F} \approx e_{F}$ $=k_{F}^{2} / 2 m_{F}=2 \pi \rho_{F} / m_{F}$ and $\mu_{B}$. A fermion-boson mixture is an experimentally relevant situation, because at low density and temperature the most important contribution to the scattering amplitude is due to $s$-wave collisions which, in the case of spin-polarized fermions, are forbidden by Pauli's principle. As a consequence, it is difficult to cool a sample of spin-polarized fermionic atoms to reach the temperatures needed to observe quantum degeneracy. This problem may be overcome by sympathetically cooling the fermions with a gas of bosons, so that the fermions cool down by interacting with the bosons [12-14].

We assume in the following the idealized zerotemperature case. The energy gaps characterizing the pairing can always be converted into critical temperatures by multiplying with the factor $\gamma / \pi \approx 0.567$ as in three dimensions [15]. We also assume $\mu_{B} \ll \mu_{F}$, which will be justified later.

Pairing in two dimensions has the peculiar feature that, for an attractive $s$-wave interaction between two different fermionic species, a bound state (of binding energy $E_{b}$ ) is always present and therefore the system enters the strongcoupling regime at sufficiently low density [9,16-18], forming a Bose condensate of fermion pairs characterized by

$$
\begin{gathered}
\mu_{F} \rightarrow e_{F}-E_{b} / 2, \\
\Delta_{0} \rightarrow \sqrt{2 E_{b} e_{F}},
\end{gathered}
$$

where $\Delta_{0}$ is the $s$-wave pairing gap. This strong pairing contrasts with the $3 \mathrm{D}$ weak-coupling behavior, where the gap vanishes exponentially with $k_{F} \rightarrow 0$. Moreover, the strongcoupling situation implies that the pairing gap is quite insensitive to an asymmetry in the population of the two species [19], contrary to the 3D case, where even a minute excess of particles of one species reduces considerably the gap size due to the effects of Pauli blocking in the gap equation $[4,20]$.

We therefore exclude in the following this "trivial" case, and focus on the situation where $s$-wave pairing is not possible, either due to a repulsive $s$-wave interaction, or when treating a system of identical (spin-polarized) fermions. The next possibility concerns the $p$-wave pairing gap, $\Delta_{1}$ $\equiv \Delta_{L=1}\left(k_{F}\right)$, which in the low-density limit is given by the weak-coupling result [18]

$$
\frac{\Delta_{1}}{\mu_{F}}=c_{1} \exp \left[-\frac{2 \pi}{m_{F} T_{F}}\right],
$$

where $c_{1}$ is a constant of order unity and

$$
\begin{gathered}
T_{F}=T_{k_{F} k_{F}}^{(L=1)}\left(2 \mu_{F}\right)=\int_{0}^{\pi} \frac{d \boldsymbol{\phi}}{\pi} \cos \phi\left\langle\boldsymbol{k}^{\prime}\left|T\left(2 \mu_{F}\right)\right| \boldsymbol{k}\right\rangle, \\
|\boldsymbol{k}|=\left|\boldsymbol{k}^{\prime}\right|=k_{F}, \quad \cos \phi=\hat{\boldsymbol{k}}^{\prime} \cdot \hat{\boldsymbol{k}}
\end{gathered}
$$

is the relevant $T$-matrix element of the interaction.

We now analyze the pairing force mediated by the surrounding bosons. Assuming for the moment that a direct fermion-fermion interaction is absent, the relevant interaction to leading order in density, to be used in Eq. (2), is $T_{F}$ $=\Gamma_{F}$, where $\Gamma_{F}$ is the boson-mediated irreducible polarization interaction, schematically represented in Fig. 1. Within the range of the weak-coupling formula, it is sufficient to consider incoming and outgoing fermions on the Fermi sur- 
a)

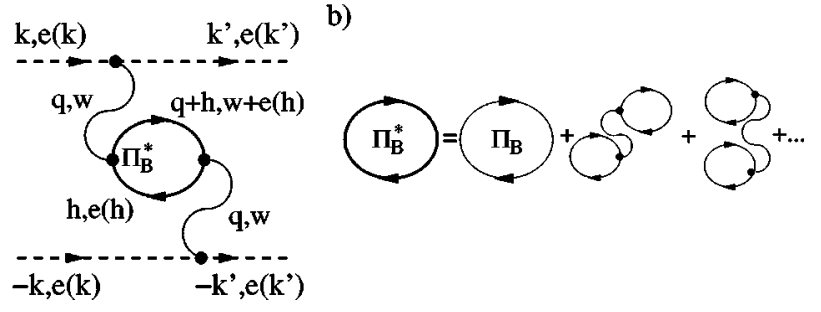

FIG. 1. (a) Polarization interaction $\Gamma$ between two fermions (dashed lines) mediated by the presence of bosons (solid lines). The labels indicate the momentum and energy of each line. For condensate bosons and fermions on the Fermi surface, $\boldsymbol{h}=\mathbf{0}, \omega=0$. (b) Diagrams contributing to the boson bubble in RPA; the last one is an example of a backward-going diagram, negligible when $\mu_{B}$ $\rightarrow 0$. Here, thick solid lines are full propagators, thin solid lines are free propagators, and wiggles represent interactions.

face and on their energy shell, cf. Eq. (3). Therefore, the energy transfer from one fermion to the other vanishes: $\omega$ $=0$. For the time being we assume for simplicity bosonfermion $(B F)$ and boson-boson $(B B) T$ matrices that can be considered constant in the low-density limit, as in the threedimensional case. In two dimensions this is, however, not anymore true $[21,22]$, and the correct treatment will be discussed further below.

With this assumption, the relevant interaction kernel reads at low density [7]

$$
\left\langle\boldsymbol{k}^{\prime}\left|\Gamma_{F}\right| \boldsymbol{k}\right\rangle=T_{B F}^{2} \Pi_{B}^{*}\left(\left|\boldsymbol{k}^{\prime}-\boldsymbol{k}\right|\right),
$$

with the bosonic RPA propagator

$$
\Pi_{B}^{*}(q)=\frac{\Pi_{B}(q)}{1-T_{B B} \Pi_{B}(q)}
$$

and the bosonic static Lindhard function

$$
\Pi_{B}(q)=-\frac{4 m_{B} \rho_{B}}{q^{2}} .
$$

We have neglected the influence of the fermions on the properties of the Bose condensate. We remark at this point that due to the $1 / q^{2}$ dependence of the two-dimensional Lindhard function, the RPA has necessarily to be performed in order to avoid divergencies. The situation is similar to the electron gas where, however, the interaction is singular.

Projecting out the $L=1$ partial-wave $F F$ interaction, one obtains in particular

$$
\begin{gathered}
\Gamma_{k_{F} k_{F}}^{(L=1)}=T_{B F}^{2} \int_{0}^{\pi} \frac{d \phi}{\pi} \cos \phi \Pi_{B}^{*}\left[q=\sqrt{2(1-\cos \phi)} k_{F}\right] \\
=-T_{B F}^{2} \frac{x}{T_{B B}} \int_{0}^{\pi} \frac{d \phi}{\pi} \frac{\cos \phi}{x+1-\cos \phi}=-\frac{T_{B F}^{2}}{T_{B B}} g(x), \\
x=\frac{2 m_{B} T_{B B} \rho_{B}}{k_{F}^{2}}=\frac{m_{B} T_{B B}}{2 \pi} \frac{\rho_{B}}{\rho_{F}},
\end{gathered}
$$

with

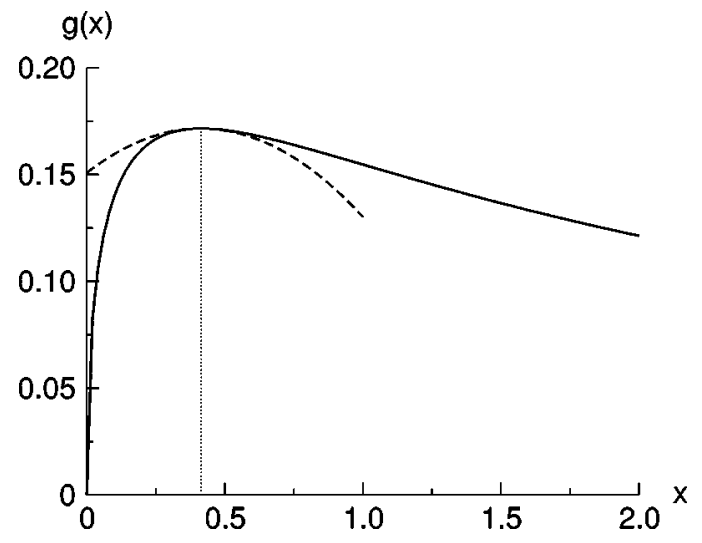

FIG. 2. The function $g$ appearing in Eq. (8) (solid line), together with the parabolic approximation (dashed line) around its maximum (indicated by the dotted vertical line).

$$
g(x)=\frac{1+x}{\sqrt{1+2 / x}}-x .
$$

This function is plotted in Fig. 2. It has a maximum located at $(x=\sqrt{2}-1 \approx 0.414, g=3-2 \sqrt{2} \approx 0.172)$, and can in its vicinity be approximated by a parabola, as shown by the dashed line in the figure. This translates into a sharp Gaussian peak for the gap function, according to Eq. (2).

Therefore, when increasing the boson density for fixed $\rho_{F}$, the induced fermionic attraction and thus also the pairing gap would reach a maximum for

$$
\frac{\rho_{B}}{\rho_{F}}=\frac{0.414 \times 2 \pi}{m_{B} T_{B B}} .
$$

However, in two dimensions the ( $s$-wave) scattering matrices $T_{B F}$ and $T_{B B}$ cannot be considered constant, but vanish logarithmically with the center-of-mass system (c.m.s.) energy $E$ of the two-particle state [21,22], i.e.,

$$
\left\langle\boldsymbol{k}^{\prime}|T(\boldsymbol{P}=\mathbf{0}, E \rightarrow 0)| \boldsymbol{k}\right\rangle \rightarrow \frac{2 \pi}{m} \frac{1}{\ln \left(E_{0} /|E|\right)}
$$

where $m$ is the reduced mass of the colliding particles and $E_{0} \gg E$ is a parameter (with dimensions of energy) characterizing low-energy scattering. Therefore, it is necessary to evaluate the c.m.s. energy $E^{2}=P_{\mu} P^{\mu}$ for the following situations (sketched in Fig. 3):
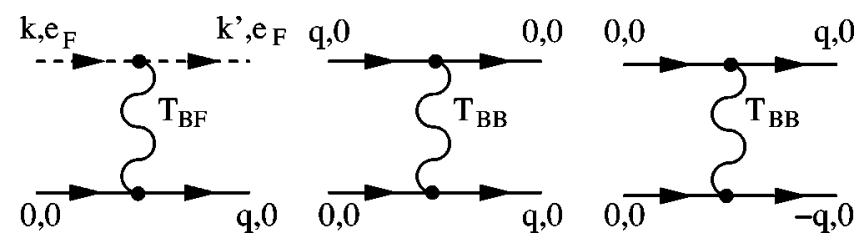

FIG. 3. Possible collision events in the mixture, according to Eq. (11). Dashed lines denote fermions, solid lines bosons, and wiggles represent interactions. The labels indicate the momentum and energy of each particle. 


$$
\begin{array}{r}
\left\langle\left(\boldsymbol{k}^{\prime}, \mu_{F}\right)(\boldsymbol{q}, 0)\left|T_{B F}\right|\left(\boldsymbol{k}, \mu_{F}\right)(\mathbf{0}, 0)\right\rangle: E=\frac{k_{F}^{2}}{2 m_{F}} \frac{m_{B F}}{m_{F}}, \\
\left\langle(\mathbf{0}, 0)(\boldsymbol{q}, 0)\left|T_{B B}\right|(\boldsymbol{q}, 0)(\mathbf{0}, 0)\right\rangle: E=-\frac{q^{2}}{4 m_{B}}, \\
\left\langle(+\boldsymbol{q}, 0)(-\boldsymbol{q}, 0)\left|T_{B B}\right|(\mathbf{0}, 0)(\mathbf{0}, 0)\right\rangle: E=0,
\end{array}
$$

with $m_{B F}=m_{B} m_{F} /\left(m_{B}+m_{F}\right)$ the $B F$ reduced mass. Therefore, within the approximation $\mu_{B}=0$ (or more precisely $\mu_{B} \ll \mu_{F}$ ), only forward-going polarization diagrams [see Fig. 1(b)] contribute to the induced $F F$ interaction. This can be taken into account by replacing $T_{B B} \Pi_{B}(q)$ $\rightarrow T_{B B}(q) \Pi_{B}(q) / 2$ in Eq. (5), where now

$$
T_{B B}(q)=\frac{4 \pi}{m_{B}} \frac{1}{\ln \left(4 m_{B} E_{B B} / q^{2}\right)} .
$$

Also, the relevant boson-fermion interaction becomes

$$
T_{B F}\left(k_{F}\right)=\frac{2 \pi}{m_{B F}} \frac{1}{\ln \left(2 m_{F}^{2} E_{B F} / m_{B F} k_{F}^{2}\right)} .
$$

Here $E_{B F}$ and $E_{B B}$ are the parameters characterizing lowenergy $s$-wave $B F$ and $B B$ scattering, respectively.

We obtain then

$$
\begin{gathered}
\Gamma_{k_{F} k_{F}}^{(L=1)}=-\frac{m_{B} T_{B F}^{2}\left(k_{F}\right)}{2 \pi} h(x, y), \\
h(x, y)=\int_{0}^{\pi} \frac{d \phi}{\pi} \frac{\cos \phi}{(1-\cos \phi) / x-1 / \ln [(1-\cos \phi) / y]}, \\
x=\frac{4 \pi \rho_{B}}{k_{F}^{2}}=\frac{\rho_{B}}{\rho_{F}}, \quad y=\frac{m_{B} E_{B B}}{m_{F} \mu_{F}},
\end{gathered}
$$

with the condition $y \gg 1$ for Eq. (12) to be valid.

Varying the boson density (i.e., $x$ ) for a constant fermion density $(y)$, one observes again a maximum at a certain ratio $x_{\text {opt }}(y)$. The optimal ratio $x_{\text {opt }}$ as well as the corresponding value $h_{\mathrm{opt}}$ are plotted in Fig. 4 as functions of $y$. At sufficiently large $y$ one obtains a quasilinear dependence on $\ln y$ :

$$
\begin{aligned}
& x_{\text {opt }}(y) \rightarrow 0.414(3.0+\ln y), \\
& h_{\text {opt }}(y) \rightarrow 0.172(3.7+\ln y) .
\end{aligned}
$$

We remark that in fact the optimal ratio $x_{\text {opt }}$ corresponds to the one for a constant $T_{B B}$, Eq. (9), when making the replacement

$$
T_{B B} \rightarrow \frac{T_{B B}\left(q=0.317 k_{F}\right)}{2} .
$$

Thus position and value of the maximum depend logarithmically on the Fermi momentum. Taking all these facts into account, the value of the pairing gap under optimal conditions becomes

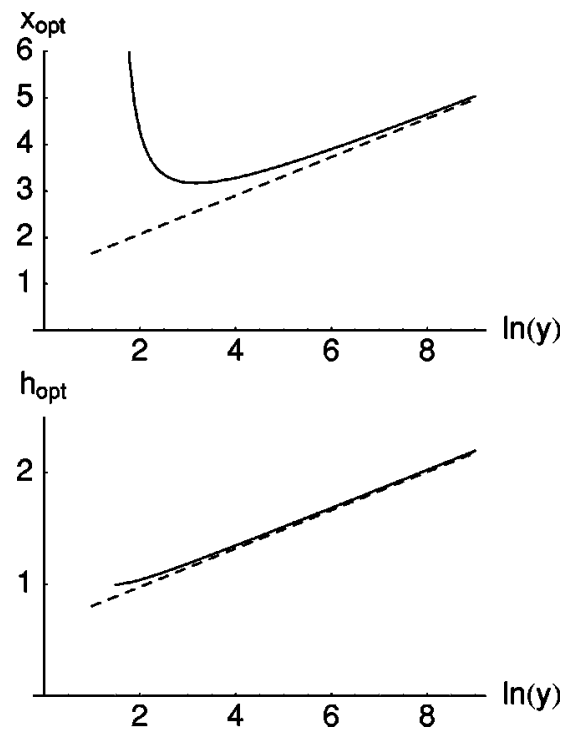

FIG. 4. The optimal values $x_{\mathrm{opt}}$ and $h_{\mathrm{opt}}$ for the pairing interaction, Eq. (14). The dashed lines indicate the asymptotic behavior, Eq. (15).

$$
\ln \frac{\Delta_{1}}{c_{1} \mu_{F}} \rightarrow-\frac{m_{B F}^{2}}{m_{B} m_{F}} \frac{\left[\ln \left(m_{F} E_{B F} / m_{B F} \mu_{F}\right)\right]^{2}}{0.172[3.7+\ln y]}
$$

The induced interaction, Eq. (14), is to be compared with the direct low-density $p$-wave fermion-fermion interaction [18],

$$
T_{k_{F} k_{F}}^{(L=1)}\left(2 \mu_{F}\right) \approx \frac{4}{m_{F}} \frac{\mu_{F}}{E_{1}} \sim \rho_{F},
$$

where $E_{1}$ is the parameter characterizing 2D low-density $p$-wave scattering. Therefore, at sufficiently low fermion density, the boson-mediated attraction, Eqs. (14) and (15), becomes dominant, since it depends only logarithmically on the fermion density. For the same reason, any fermionic polarization corrections have also been neglected.

We analyze finally the assumption $\mu_{B} \ll \mu_{F}$ that was made beforehand. The boson chemical potential is determined by $[23,24]$

$\mu_{B}=\rho_{B} T_{B B}\left(E=\alpha \mu_{B}\right)=\frac{4 \pi \rho_{B}}{m_{B}} \frac{1}{\ln \left(E_{B B} / \alpha \mu_{B}\right)} \ll \mu_{F}=\frac{2 \pi \rho_{F}}{m_{F}}$,

where $\alpha$ is of order unity [28]. Since the logarithm in the low-density domain is always large, we have the sufficient condition

$$
x=\rho_{B} / \rho_{F} \lesssim m_{B} / m_{F} .
$$

In order to estimate typical sizes of the expected gap, we plot in Fig. 5 the gap $\Delta_{1} / \mu_{F}$, according to Eq. (17), as a function of the ratios $\mu_{F} / E_{B B}$ and $\mu_{F} / E_{B F}$ (assuming for 


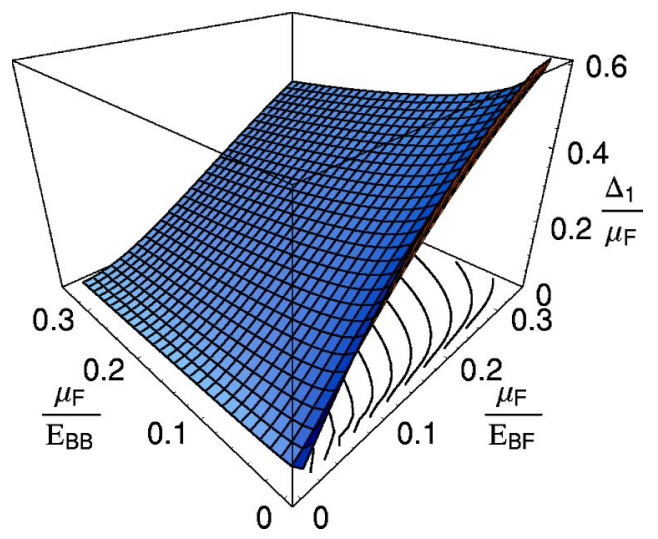

FIG. 5. The pairing gap for optimal boson concentration, Eq. (17), as a function of the fermion chemical potential.

simplicity $c_{1}=1$ and $m_{F}=m_{B}$ ). One notes that it is mainly the ratio $\mu_{F} / E_{B F}$ that determines the gap, whereas the dependence on $\mu_{F} / E_{B B}$ is relatively weak. Thus with fermion chemical potentials $\mu_{F} \lesssim E_{B F}$ quite large gaps $\Delta_{1} \lesssim \mu_{F}$ could be achieved.

In order to translate this condition into experimental quantities, we use the results of Refs. [8,24], relating the 2D scattering parameter $E_{B F}$ to the value of the $3 \mathrm{D}$ scattering length $a_{B F}$, for a boson-fermion system confined in a strongly anisotropic trap characterized by frequencies $\omega_{\perp}$ and $\omega_{z}$ (here supposed to be the same for bosons and fermions), obtaining

$$
\frac{\mu_{F}}{E_{B F}}=\frac{\pi}{B} \frac{\mu_{F}}{\omega_{z}} \exp \left(-\sqrt{2 \pi} \frac{l_{z}}{a_{B F}}\right),
$$

where $B \approx 0.915$ and $l_{z}=1 / \sqrt{2 m_{B F} \omega_{z}}$. Since at the same time for a $2 \mathrm{D}$ situation the condition $\mu_{F} \ll \omega_{z}$ must be fulfilled, one can only expect observable gaps if the exponential term is not too small. One can now distinguish two cases.

(i) $a_{B F}>0$. In this case the ratio $l_{z} / a_{B F}$ should be minimized as much as possible, i.e., for extremely strongly $z$-compressed traps, or for systems with a very large BF scattering length (Feshbach resonance).

(ii) $a_{B F}<0$. In this case the exponential term is never small and observable pairing can be expected provided the ratio $\mu_{F} / \omega_{z}$ is not too small. Using the Thomas-Fermi approximation $\mu_{F}=\sqrt{2 N_{F}} \omega_{\perp}$ for the chemical potential of a two-dimensional Fermi gas in a (in-plane) harmonic trap of frequency $\omega_{\perp}$, this last condition can be expressed by means of the fermion number and the trap asymmetry:

$$
\frac{\mu_{F}}{\omega_{z}}=\frac{\omega_{\perp}}{\omega_{z}} \sqrt{2 N_{F}}
$$

Thus under favorable circumstances quite large $p$-wave pairing gaps of the order of the Fermi energy seem to be achievable, comparable to those of $s$-wave pairing in quasi-2D twocomponent Fermi gases [9]. Unfortunately, more precise quantitative predictions cannot be made in this regime, since with $\mu_{F} \approx E_{B F}, E_{B B}$ also the asymptotic expression Eq. (10) becomes invalid. It is worth noticing that the same effect in three dimensions is less effective in increasing the size of the gap, and one expects $\Delta_{1} / \mu_{F} \leqslant 0.1$ [5].

Finally, we consider the problem of the phase stability of boson-fermion mixtures, which has been faced by different authors $[6,25,26]$ that reach similar conclusions. This problem has been studied for homogeneous as well as for trapped systems, but always in three dimensions, where the theoretical description is somehow easier than in two dimensions because of the different behavior of the corresponding $T$ matrices at low energy. Here we briefly discuss the implications from the previous studies $[6,25,26]$ that can be applied to our case, but a more precise analysis would be of high interest.

According to Ref. [6], in a boson-fermion mixture one can expect to find one of three situations: (i) a fermionic phase and a bosonic phase, (ii) a fermionic phase and a boson-fermion mixture, and (iii) a single uniform mixture. In case (i) there is no boson-fermion induced interaction and no sympathetic cooling. In case (ii) these problems are overcome, but only a fraction of the fermions is efficiently cooled and can undergo the superfluid transition. Therefore, the interesting situation is that of case (iii). This can be obtained if there is attraction between bosons and fermions (to avoid their spatial separation), but in this case the system may collapse due to this same attraction [25]. This will happen if, e.g., the number of bosons exceeds some critical number $N_{\mathrm{cr}}$, which will depend on $a_{B B}$ and $a_{B F}$. For a uniform system, we know that $a_{B B}>0$ is required in order to avoid the collapse of the boson component. This also stabilizes significantly the mixtures [25], even for $a_{B F}<0$. As expected, the case $a_{B F}>0$ rapidly gives rise to spatial separation of the two gases [25].

Applying these arguments to the mixtures used in typical experiments, we see that the case ${ }^{7} \mathrm{Li}-{ }^{6} \mathrm{Li}$ (where the assumption $m_{B}=m_{F}$ is more adequate) with $a_{B B}=-1.5 \mathrm{~nm}$ and $a_{B F}=2.2 \mathrm{~nm}$ [13] does not correspond to the optimal stability conditions. However, the presence of the trapping stabilizes the system so that experiments can be performed. On the other hand, for the ${ }^{87} \mathrm{Rb}-{ }^{40} \mathrm{~K}$ mixture, where $a_{B B}$ $=5.2 \mathrm{~nm}[14]$ and $a_{B F}=-2.2 \mathrm{~nm}$ [27], the stability conditions for the homogeneous case are fully satisfied.

In conclusion, we have studied the characteristics of $p$-wave pairing in a two-dimensional boson-fermion mixture with repulsive (or absent) $F F s$-wave interaction. The bosoninduced attraction between two fermions dominates at low density an eventual direct $F F p$-wave force. The induced pairing gap becomes maximal at a certain optimal bosonfermion ratio. In contrast to the three-dimensional case, this ratio itself increases when decreasing the fermion density, due to the logarithmic energy dependence of the $B B T$ matrix at low density. Using this optimal condition, we have estimated the size of the gap and find experimentally achievable values, in particular for systems with a negative bosonfermion scattering length such as the ${ }^{87} \mathrm{Rb}-{ }^{40} \mathrm{~K}$ mixture.

We would like to acknowledge valuable discussions with M. Guilleumas and M.A. Baranov. This work was supported in part by the program DGICYT (Spain) No. BFM200201868. J. M.-P. acknowledges support from the Generalitat de Catalunya. 
[1] B. DeMarco and D.S. Jin, Science 285, 1703 (1999).

[2] H.T.C. Stoof, M. Houbiers, C.A. Sackett, and R.G. Hulet, Phys. Rev. Lett. 76, 10 (1996); M. Houbiers, R. Ferwerda, H.T.C. Stoof, W.I. McAlexander, C.A. Sackett, and R.G. Hulet, Phys. Rev. A 56, 4864 (1997).

[3] M.Yu. Kagan and A.V. Chubukov, Pis'ma Zh. Eksp. Teor. Fiz. 47, 525 (1988) [JETP Lett. 47, 614 (1988)]; M.A. Baranov, Yu. Kagan, and M.Yu. Kagan, ibid. 64, 273 (1996) [ibid. 64, 301 (1996)].

[4] J. Mur-Petit, A. Polls, and H.-J. Schulze, Phys. Lett. A 290, 317 (2001).

[5] H. Heiselberg, C.J. Pethick, H. Smith, and L. Viverit, Phys. Rev. Lett. 85, 2418 (2000).

[6] L. Viverit, C.J. Pethick, and H. Smith, Phys. Rev. A 61, 053605 (2000).

[7] M.J. Bijlsma, B.A. Heringa, and H.T.C. Stoof, Phys. Rev. A 61, 053601 (2000); D.V. Efremov and L. Viverit, Phys. Rev. B 65, 134519 (2002); L. Viverit, Phys. Rev. A 66, 023605 (2002).

[8] D.S. Petrov, M. Holzmann, and G.V. Shlyapnikov, Phys. Rev. Lett. 84, 2551 (2000); D.S. Petrov and G.V. Shlyapnikov, Phys. Rev. A 64, 012706 (2001).

[9] D.S. Petrov, M.A. Baranov, and G.V. Shlyapnikov, Phys. Rev. A 67, 031601 (2003).

[10] Yu. Kagan, V.A. Kashurnikov, A.V. Krasavin, N.V. Prokof'ev, and B.V. Svistunov, Phys. Rev. A 61, 043608 (2000); A.Yu. Cherny and A.A. Shanenko, Phys. Rev. E 64, 027105 (2001); J.O. Andersen and H. Haugerud, Phys. Rev. A 65, 033615 (2002); U. Al Khawaja, J.O. Andersen, N.P. Proukakis, and H.T.C. Stoof, ibid. 66, 013615 (2002); K.K. Das, ibid. 66, 053612 (2002); G. Huang, V.A. Makarov, and M.G. Velarde, ibid. 67, 023604 (2003); H. Saito and M. Ueda, Phys. Rev. Lett. 90, 040403 (2003).

[11] A.I. Safonov, S.A. Vasilyev, I.S. Yasnikov, I.I. Lukashevich, and S. Jaakkola, Phys. Rev. Lett. 81, 4545 (1998); H. Gauck, M. Hartl, D. Schneble, H. Schnitzler, T. Pfau, and J. Mlynek, ibid. 81, 5298 (1998); A. Görlitz, J.M. Vogels, A.E. Leanhardt, C. Raman, T.L. Gustavson, J.R. Abo-Shaeer, A.P. Chikkatur, S. Gupta, S. Inouye, T. Rosenband, and W. Ketterle, ibid. 87, 130402 (2001); I. Bouchoule, M. Morinaga, C. Salomon, and D.S. Petrov, Phys. Rev. A 65, 033402 (2002); M. Hammes, D. Rychtarik, B. Engeser, H.-C. Nägerl, and R. Grimm, Phys. Rev. Lett. 90, 173001 (2003); Y. Colombe, D. Kadio, M. Olshanii, B. Mercier, V. Lorent, and H. Perrin, J. Opt. B: Quantum Semiclassical Opt. 5, 155 (2003); G. Modugno, F. Ferlaino, R. Heidemann, G. Roati, and M. Inguscio, Phys. Rev. A 68, 011601 (2003).

[12] L. Spitzer, Physics of Fully Ionized Gases (Interscience, New
York, 1962); D.J. Larson, J.C. Bergquist, J.J. Bollinger, W.M. Itano, and D.J. Wineland, Phys. Rev. Lett. 57, 70 (1986); C.J. Myatt, E.A. Burt, R.W. Ghrist, E.A. Cornell, and C.E. Wieman, ibid. 78, 586 (1997); F.A. van Abeelen, B.J. Verhaar, and A.J. Moerdijk, Phys. Rev. A 55, 4377 (1997); F. Schreck, L. Khaykovich, K.L. Corwin, G. Ferrari, T. Bourdel, J. Cubizolles, and C. Salomon, Phys. Rev. Lett. 87, 080403 (2001); C. Presilla and R. Onofrio, ibid. 90, 030404 (2003).

[13] A.G. Truscott, K.E. Strecker, W.I. McAlexander, G.B. Partridge, and R.G. Hulet, Science 291, 2570 (2001).

[14] G. Modugno, G. Roati, F. Riboli, F. Ferlaino, R.J. Brecha, and M. Inguscio, Science 297, 2240 (2002).

[15] J.R. Schrieffer, Theory of Superconductivity (Addison-Wesley, Redwood City, 1988).

[16] S. Schmitt-Rink, C.M. Varma, and A.E. Ruckenstein, Phys. Rev. Lett. 63, 445 (1989).

[17] M. Randeria, Ji-Min Duan, and Lih-Yir Shieh, Phys. Rev. Lett. 62, 981 (1989).

[18] M. Randeria, Ji-Min Duan, and Lih-Yir Shieh, Phys. Rev. B 41, 327 (1990).

[19] U. Lombardo, P. Nozières, P. Schuck, H.-J. Schulze, and A. Sedrakian, Phys. Rev. C 64, 064314 (2001).

[20] T. Alm, B.L. Friman, G. Röpke, and H. Schulz, Nucl. Phys. A 551, 45 (1993); A. Sedrakian, T. Alm, and U. Lombardo, Phys. Rev. C 55, 582 (1997); A. Sedrakian and U. Lombardo, Phys. Rev. Lett. 84, 602 (2000).

[21] S.K. Adhikari, Am. J. Phys. 54, 362 (1986).

[22] S.A. Morgan, M.D. Lee, and K. Burnett, Phys. Rev. A 65, 022706 (2002).

[23] M. Schick, Phys. Rev. A 3, 1067 (1971); D.S. Fisher and P.C. Hohenberg, Phys. Rev. B 37, 4936 (1988); H.T.C. Stoof and M. Bijlsma, Phys. Rev. E 47, 939 (1993).

[24] M.D. Lee, S.A. Morgan, M.J. Davis, and K. Burnett, Phys. Rev. A 65, 043617 (2002).

[25] R. Roth and H. Feldmeier, Phys. Rev. A 65, 021603 (2002).

[26] K. Mølmer, Phys. Rev. Lett. 80, 1804 (1998); T. Miyakawa, T. Suzuki, and H. Yabu, Phys. Rev. A 64, 033611 (2001); X.X. Yi and C.P. Sun, ibid. 64, 043608 (2001); R. Roth, ibid. 66, 013614 (2002); L. Viverit and S. Giorgini, ibid. 66, 063604 (2002); H. Pu, W. Zhang, M. Wilkens, and P. Meystre, Phys. Rev. Lett. 88, 070408 (2002); C.P. Search, H. Pu, W. Zhang, and P. Meystre, Phys. Rev. A 65, 063615 (2002).

[27] E.G.M. van Kempen, S.J.J.M.F. Kokkelmans, D.J. Heinzen, and B.J. Verhaar, Phys. Rev. Lett. 88, 093201 (2002).

[28] In the current literature there is no agreement on the precise value of the constant $\alpha$, see Refs. [23,24]. For our purpose, this is, however, not relevant. 